\title{
La política social en situaciones de crisis generalizada e incertidumbre en Venezuela
}

\author{
Gutiérrez Briceño, Thais*
}

\section{Resumen}

En el presente trabajo se realiza un análisis de la política social implantada en Venezuela desde 1989, en el marco de los procesos de ajuste estructural, con el objetivo de establecer la relación política social-legitimidad. Se definen dos sub-períodos, a los efectos identificados: 1989-1998 y 1999-2001. Estos corresponden, respectivamente, con la profundización de la situación de crisis en el país y la redefinición que se registra en las orientaciones de la política social y económica, a partir de la aplicación de las políticas de tendencia neoliberal; $y$, con las acciones sociales que se desarrollan actualmente como parte de la construcción del nuevo proyecto de país, denominado V República. Se concluye que en ambos períodos la política social está desvinculada de una estrategia de desarrollo a mediano y largo plazo y, mantiene un carácter coyuntural, compensatorio y focalizado.

Palabras clave: Política social, legitimidad, programas de ajuste estructural.

\section{Social Policy in Times of Generalized Crisis and Uncertainty in Venezuela}

\begin{abstract}
In this paper we undertake an analysis of social policy implemented in Venezuela since 1989 within the framework of structural adjustment processes, and for the purpose of establishing a social legitimacy policy relationship. Two sub-periods are defined for this purpose, 1989-1998 and 1999-2001. These periods correspond respectively to the period of deepening crisis within the country with its redefinition registered in political, social and economic orientations in the country due to the application of neo-liberal policy, and to the social actions developed recently as part of the construction of a new national project called the V Republic. The conclusion is that in both periods social policy is unrelated to a middle and long-run development strategy. The focus is short-run crisis oriented, compensatory and focalized.
\end{abstract}

Key words: Social policy, legitimacy, structural adjustment programs.

Recibido: 02-01-08 . Aceptado: 02-03-22

Socióloga. Profesora e Investigadora de la Facultad de Ciencias Económicas y Sociales de la Universidad del Zulia. E-mail: thagu@telcel.net.ve 


\section{Introducción}

En los últimos años el deterioro del nivel de vida de la población venezolana se ha evidenciado en el incremento de los niveles de pobreza. Esta situación ha potenciado la preocupación, a nivel de diversos círculos y actores sociales (políticos, económicos, sociales e intelectuales), por el conjunto de lineamientos y acciones a través de los cuales se interviene el ámbito social, que engloban lo que se conoce como políticas sociales. Ello por los elevados costos políticos y económicos que genera la deuda social acumulada para el mantenimiento del orden societal establecido.

En este escenario -que ha traído como consecuencia la resignificación de la cuestión social-, el presente trabajo tiene como objetivo analizar la política social implantada en Venezuela desde 1989, fecha en la cual se introducen cambios en la estrategia de desarrollo a seguir, con la implantación de un programa de ajuste estructural, y, para el caso que nos interesa, nuevas orientaciones en la concepción de la política social.

A los fines de hacer más visibles los virajes a los que se ha hecho referencia; así como, para permitir la contrastación de los cambios, el artículo se inicia con una reflexión sobre la sociedad venezolana durante el período 1979-1989, desta- cándose aspectos vinculados con la relación Estado-política-economía-sociedad.

\section{Reflexiones sobre la crisis, la legitimidad y la política social (1979-1989)}

Hasta comienzos de los años ochenta la democracia venezolana lucía como una de las más estables del continente. A fines de esa década comienzan a manifestarse los primeros síntomas de su inestabilidad, los cuales indicaban que el modelo de desarrollo y de hegemonía sobre el cual se sustentó el orden democrático no respondió más a las demandas y exigencias de los distintos sectores sociales, dado los cambios ocurridos en la sociedad (como se verá más adelante).

Como históricamente es sabido, el régimen democrático representativo surgido en Venezuela a partir de 1958, tuvo como base un conjunto de compromisos y acuerdos entre distintas fuerzas sociales, conformándose un "pacto estatal" como soporte básico de dicho modelo sociopolítico. El Estado surge como integrante y garante del pacto; además de cumplir con las funciones clásicas de un Estado ligado estrechamente a la acumulación de capital, asumió múltiples funciones: productor directo, regulador de las relaciones entre el capital y el trabajo, suministrador de servicios, entre otras. Este

1 Dentro de ese conjunto de compromisos y acuerdos, el Pacto de Punto Fijo, en particular, logró estructurar en un mismo proceso un sistema y un gobierno, como dos aspectos diferenciados, en los que el sistema no se confundía con el gobierno. Esta situación quedó evidenciada el 4 de febrero de 1992, con el intento de golpe militar, éste fue contra el gobierno, el sistema democrático como valor político cultural quedó indemne. Sobre el tema, ver Arenas (1992). 
pacto colocó al Estado en el centro de las relaciones socioeconómicas y como interlocutor principal de las demandas de las distintas fuerzas y grupos sociales.

Las reglas de juego del ordenamiento socio-político sustentadas en el conjunto de acuerdos tácitos y explícitos entre las fuerzas involucradas en el "pacto estatal”: partidos políticos (Acción Democrática, $\mathrm{AD}$; Comité de Organización Político Electoral Independiente, COPEI y Unión Republicana Democrática, URD), sector empresarial (Federación de Cámaras y Asociaciones de Comercio, Fedecámaras), sector sindical (Confederación de Trabajadores de Venezuela, CTV), Fuerzas Armadas e Iglesia, definieron las reglas de convivencia y el modo de conciliación de intereses entre las elites pactantes. Esta situación estableció los límites de la legitimidad.

Tal y como lo plantean algunos autores (Rey, 1989 y Kornblith, 1994), la conciliación de intereses fue posible por: 1) la existencia de recursos provenientes de la renta petrolera -factor fundamental en este proceso-, los cuales permitieron al Estado atender las demandas sociales de las diferentes fuerzas sociopolíticas sin generar conflictos redistributivos; 2 ) el nivel de expectativas sociales fue posible satisfacerlo con los recursos existentes; y 3) gracias a la representatividad de las organizaciones (partidos políticos y sindicatos) para agregar y canalizar las demandas.
En este proceso de negociaciones y acuerdos se definió el modelo de desarrollo económico y social del país; así como, las reglas de la representación democrática y, las cuotas de poder y participación de las fuerzas comprometidas en la implantación del proyecto socio-político propuesto.

En lo que se refiere a la cuestión social, el modelo democrático se erigió sobre la base de impulsar el bienestar social a través de la política estatal. La renta petrolera fue el factor que permitió que el Estado cumpliera con las funciones de acumulación y legitimación: crear las condiciones para la acumulación del capital y, mantener la armonía social y el reconocimiento de la población al régimen. Ello, por la vía de redistribuir la renta a la sociedad. Esta situación permitió el establecimiento de un Estado DesarrollistaPopulista. El Estado fue el motor del desarrollo $y$, al mismo tiempo, proveedor de bienestar y suplidor de las expectativas y demandas sociales. Este modelo "híbrido" logró el desarrollo de dos procesos: la ciudadanización de las masas y la socialización del consumo, lo cual hizo posible la implantación de un modelo "exitoso" 2 que conjugó democracia con crecimiento, como nunca antes había tenido lugar en América Latina.

En este marco, la política social implantada fue de corte y orientación universalista, dirigida a la prestación de servicios básicos a la población, destacó por

2 El calificativo de exitoso refiere únicamente a la posibilidad de apuntalar el crecimiento económico al mismo tiempo que podía satisfacer las expectativas y demandas de los diversos sectores sociales, de una forma populista. En ningún momento a los resultados de un balance con respecto al desarrollo del país. 
la gratuidad y acceso creciente a la educación y al servicio de salud; así como, por el predominio de políticas de subsidios directos e indirectos y el control de precios. El acceso por parte de la población a los bienes y servicios brindó a amplios sectores sociales la posibilidad de movilidad social e integración social, con lo cual sus expectativas pudieron ser satisfechas.

Es de destacar, que la magnitud de los recursos del Estado tuvo como consecuencia que, en lo atinente a la gestión de las instituciones de prestación de servicios sociales, el énfasis se dirigiera hacia la eficacia de las acciones y no hacia el uso eficiente de los recursos utilizados. Al respecto señala Piñango (1999:116): "Poco importaba, por ejemplo, si se estaba subsidiando la alimentación de quienes disponían de ingresos suficientes, con tal de que los alimentos baratos llegaran a la gente de menores ingresos".

La dinámica del desarrollo de la sociedad generó cambios en las condiciones bajo las cuales el modelo de desarrollo y el modelo de hegemonía habían funcionado eficientemente: crisis económica, complejidad y fragmentación social, cuestionamiento de los canales de representación, cambios en los patrones socio-culturales, entre otras. Así, los estallidos de violencia social del 27 y 28 de febrero de 1989 evidenciaron los primeros síntomas de la ruptura del consenso social y; los intentos de golpe de Estado del 4 de febrero y del 27 de noviembre de
1992, la ruptura del consenso político. Esto es, el agotamiento del modelo Estatal instaurado desde 1957, el cual no podía seguir satisfaciendo simultáneamente las necesidades de acumulación y legitimación de la sociedad venezolana del momento.

Diversos han sido los calificativos dados a la crisis: económica, política, de la democracia, de legitimidad, moral, ética, entre otros. En este trabajo se asume que la crisis fue sistémica ${ }^{3}$, producto de las contradicciones existentes entre las demandas de la economía y las necesidades de legitimación del orden político. Es decir, de la incapacidad del Estado para satisfacer las necesidades de políticas de autogobierno al sistema económico y las necesidades de legitimación del sistema político, que en tiempos normales podía realizar. Es de aclarar que, en lo referente a este modelo sistémico, a partir de la revisión del planteamiento de la teoría de sistemas, básicamente de Luhmann (1990) quien señala que la autorreferencialidad de los sistemas es el mecanismo por el que se producen y reproducen por sí mismos, dejando de lado el papel de la acción social y de lo normativo; y los aportes de Habermas (1975), con el propósito de hacer visible la importancia del orden normativo en los procesos de integración social.

En este sentido, se retoma el papel del sistema socio-cultural, quien proporciona los elementos de legitimidad al sistema social. Situaciones nuevas (crisis por diversos subsistemas, diferenciados e interrelacionados, en este caso, por el subsistema económico, el subsistema político-estatal y el subsistema socio-cultural. 
económica, por ejemplo) pueden producir rupturas o perturbaciones en los procesos de integración social, debido a la pérdida de los esquemas de interpretación de sentido del ordenamiento vigente aceptados como válidos, los cuales son producto de entendimientos acordados intersubjetivamente. La pérdida del sentido del orden constituido produce perturbaciones en los procesos de legitimación, pues los esquemas de interpretación tradicional ya no son válidos para enfrentar las nuevas situaciones, produciéndose eventos de anomia social.

Ante la crisis económica, cuyo inicio suele establecerse en el período comprendido entre 1978 y $1983^{4}$, el Estado no podía seguir satisfaciendo simultáneamente las demandas de la economía que facilitan la reproducción del capital y las aspiraciones de los distintos sectores sociales con los cuales asegura la lealtad de las masas y la legitimidad del sistema. En esta situación, el Estado filtra las demandas de la sociedad civil con la consecuente pérdida de apoyo de las masas y de la legitimidad. Pues, las demandas que ingresan al Estado son mayores que su capacidad para resolverlas; produciéndose dificultades en los mecanismos de generación de consenso y legitimidad. Esto rompe con los parámetros de bienestar instrumentalizados y genera problemas a nivel de la integración social y normativa.

Si bien la crisis por la que atravesó la sociedad venezolana de ese período fue una crisis generalizada, las salidas propuestas en un primer momento fueron eminentemente económicas. Estas se concretaron en la implantación de políticas de ajuste estructural, más exactamente, con la puesta en marcha del llamado "Paquete de Medidas de Ajuste

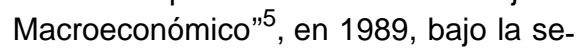

4 Sin entrar en una discusión sobre la crisis del modelo de desarrollo venezolano, se toma como fecha de inicio de la misma el año 1978, por considerar que los indicadores económicos muestran el comienzo del período de recesión económica. Sin embargo, otros autores señalan el año 1983, por ser el momento cuando se acentúa el estancamiento económico, la inflación, el desempleo, ocurre la devaluación de la moneda y se aplican controles de cambio. Al respecto, $\mathrm{Vi}-$ vancos y España (1993) plantean que, desde 1978 el crecimiento económico no era sostenible, lo que se evidenció en la caída del producto a partir de ese año, pero que no fue hasta febrero de 1983, con la devaluación de la moneda, cuando la comunidad económica internacional rehusó continuar entregando renta a futuro a Venezuela. También es interesante el planteamiento de López Maya y Lander (2000:188), quienes señalan que el llamado "viernes negro" (18-021983), fecha en la que se decreta la devaluación de la moneda, "marca de manera simbólica el despertar en la conciencia colectiva de la dimensión y gravedad de la crisis económica por la cual atravesaba el país" o, dicho en clave sociológica, significó la subjetivación de la crisis por la población venezolana. Así mismo, Portillo (1998), destaca esta misma fecha aduciendo que la crisis, cuya tipificación adquiere un carácter económico, tuvo manifestaciones que se expresaron en múltiples dimensiones de la sociedad (política, social, cultural).

5 Es de aclarar que este no fue el primer programa de ajuste estructural que se intentó implantar en el país. Durante el gobierno de Luis Herrera Campins (1979-1984), la política económica tuvo una orientación de tendencia neoliberal, la cual no fue aplicada consistentemente por el alza de los precios del petróleo en 1979-1980. Posteriormente, durante el gobierno de Jaime Lusinchi (1984-1989) se intentó la aplicación de un programa de políticas de ajuste, de corte heterodoxo, interrumpido en 1986 (Véase López Maya y Lander, 2000). 
gunda presidencia de Carlos Andrés Pérez. Estas medidas estuvieron vinculadas a los lineamientos y exigencias que los organismos financieros multilaterales, básicamente Fondo Monetario Internacional (FMI) y Banco Mundial (BM), impulsaron en América Latina en la década de los 80 , en el marco de la crisis del endeudamiento externo, como condición para la renegociación y refinanciamiento de la deuda externa.

Esta política de orientación neoliberal, persiguió regular los desequilibrios macroeconómicos y modernizar la economía y, se centró en la instauración de una economía de libre mercado, la reducción de la intervención del Estado en la economía y la transferencia a los ciudadanos de las responsabilidades económicas y sociales. Es importante tener presente, como se desprende de lo antes dicho, que la concepción neoliberal no se circunscribe únicamente a lo económico, contiene una propuesta de orden societal $^{6}$ que implica el establecimiento del mercado como eje ordenador de la sociedad y, consecuentemente, la redefinición de la relación Estado-mercado-sociedad.

Ahora bien, no puede soslayarse el impacto que en la estabilidad del orden socio-político han tenido estas políticas por cuanto se ha comprometido la capacidad del Estado para satisfacer las demandas de los diversos sectores de la po- blación y obtener así el reconocimiento y aceptación de la ciudadanía. Además, de acentuar la concentración del ingreso y elevar los niveles de pobreza de la población.

Dentro de las alternativas a la situación, y en el marco de las políticas de ajuste implementadas, la política social se perfiló como un mecanismo que apuntó a evitar la crisis de integración social producida por la pérdida de la capacidad del estado para generar consenso y legitimidad. En efecto, su objetivo se dirigió a lograr apoyos y lealtades de la población al régimen democrático para evitar la crisis de gobernabilidad y perdida de legitimidad, puesto que la orientación de los ajustes económicos conllevaría a la crisis del Estado de Bienestar. En este contexto se redefine la política social. Es de destacar que la instrumentación de la nueva política social no significó la eliminación de la totalidad de la anterior política social, sino un desplazamiento en la prioridad y el énfasis de las políticas y programas sociales ${ }^{7}$ a ejecutar.

\section{Procesos de ajuste, pobreza y política social (1989-1998)}

Como se señaló anteriormente, en el año 1989 se introducen cambios sustanciales en la política económica, ante la llegada de Carlos Andrés Pérez al poder.

6 Esta propuesta de orden societal tiene un marcado acento economicista, puesto que el mercado se convierte en el nuevo código ordenador de la sociedad. Esta centralidad otorgada a "lo económico" es indicativa del carácter subordinado con el que es tratado "lo social".

7 Lo que significó la disminución de la atención del Estado hacia áreas sociales tradicionales como salud, vivienda, educación, entre otras, y el deterioro de la calidad de la prestación de estos servicios. 
La profundización de la crisis económica y el deterioro de las condiciones sociales existentes en el país hicieron impostergables las redefiniciones efectuadas. El programa de ajuste estructural instrumentado se dirigía a enfrentar los desequilibrios internos y externos; así como, a corregir los indicadores sociales que evidenciaban la crítica situación social, específicamente, la tasa de desempleo y los niveles de pobreza.

El llamado "Paquete Económico", de naturaleza ortodoxa, anunciado el 16 de febrero de 1989, contemplaba las siguientes políticas: "a. restricción del gasto fiscal; b. restricción de los niveles salariales ; c. unificación del régimen cambiario con paridad unitaria y flotante; $d$. Tasas de interés flexibles y aumento inmediato de los niveles de las tasas de interés reguladas, eliminación de los créditos a tasas preferenciales para la agricultura, establecimiento de tasas de interés por el mercado tan pronto como fuera posible; e. reducción de los controles de precios; f. posposición de programas de inversión de baja prioridad; $g$. reducción de los subsidios; $h$. introducción de un impuesto sobre la venta; i. ajuste de las tarifas de los bienes y servicios provistos por empresas estatales, incluyendo los precios de los productos petroleros en el mercado interno; $j$. reforma en el régimen comercial, incluyendo la eliminación de la mayor parte de las excepciones en las tarifas y liberación de las importaciones; $k$. levantamiento a las restricciones de las transacciones internacionales, incluyendo la inversión extranjera y la repatriación de dividendos" (López Maya y Lander, 2000: 19).

El alza de los precios de la gasolina y del transporte, ocasionaron 11 días después los hechos de violencia social que expresaron la resistencia popular a las medidas implantadas, conocidos como los sucesos del 27 y 28 de febrero de 1989. Estos sucesos obligaron al gobierno a diseñar un conjunto de medidas sociales para contrarrestar los efectos desencadenados por las medidas económicas en la población. En este contexto se diseñó el Plan de Enfrentamiento a la Pobreza (PEP), que recoge las directrices de la nueva política social.

Entre los principales programas que contempló el Plan de Enfrentamiento a la Pobreza están: Beca Alimentaria, Beca Láctea, Beca de Cereales, Vaso de Leche Escolar, Merienda Escolar, Comedores Escolares, Lactovisoy al Escolar, Programa Alimentario Materno Infantil, Programa de Atención en Salud, Programa de Atención Comunitaria, Dotación de Uniformes y Útiles Escolares, Ampliación de la Cobertura de los Pre-escolares, Compensación Socio-Pedagógica y Cultural, Capacitación y Empleo Juvenil, Subsidio al Pasaje Preferencial Estudiantil, Hogares de Cuidado Diario, Seguro de Paro Forzoso, Programa de Apoyo a la Economía Popular, Programa Nacional de Beca Salario, Vivienda Rural, Proyecto de Mejoramiento Urbano en Barrios y Programa de Inversión Social Local (González, 1996).

Como señala Silva Michelena (1999:97): "Este Plan marcó toda la acción de su gobierno, y antecedió al VIII Plan de la Nación, que solo se diseño en 1990". En efecto, estas medidas están recogidas en el VIII Plan de la Nación (1990-1993), (CORDIPLAN, 1990), donde se plantean los lineamientos del llamado "El Gran Viraje", que expresa las modi- 
ficaciones en la política económica y social. Estas modificaciones conceptualmente representan un cambio en la estrategia de desarrollo a seguir, ante el agotamiento del modelo de desarrollo anterior.

Con las nuevas directrices de la política social se introducen cambios sustantivos: su objetivo pasa a ser la compensación de los efectos negativos de los ajustes macroeconómicos en los sectores más vulnerables de la población, resultados derivados de la distribución asimétrica de la incidencia de dichas políticas. Definiéndose como "población vulnerable" a aquellos grupos poblacionales conformados por familias de menores ingresos, mujeres embarazadas y en período de lactancia y niños en edad preescolar y escolar, en los cuales las consecuencias de la crisis y del período recesivo del ajuste pueden provocar situaciones irreversibles, a menos que se tomen medidas puntuales, dado que no pueden trasladar los costos que sobre sus ingresos tienen tales políticas. Entre las características de la política social destacan: la orientación hacia grupos meta (focalización), identificados como los más vulnerables de la población; la privatización de los servicios sociales; el carácter transitorio; la transferencia de responsabilidades a la sociedad civil y la descentralización en la prestación de los servicios sociales.

Es de hacer notar, que la focalización tiene como propósito lograr la selectividad y jerarquización de los beneficiarios de los programas sociales y una mayor eficiencia del gasto social. Con esta nueva orientación de la política social el horizonte temporal que se maneja es el corto plazo. La transitoriedad de las acciones sociales deviene del hecho de asumir que su apli- cación se mantendrá mientras duren los efectos recesivos del plan de ajuste, es decir, hasta que se produzca el crecimiento económico y la población pueda incorporarse al proceso productivo. Esto explica su desvinculación con una estrategia de desarrollo social de largo plazo. Aspecto este, que como plantean Lacabana y Maingón (1997), estuvo presente en la concepción del desarrollo que se instrumentó en América Latina hasta fines de la década del setenta.

Como señala Vilas (1995:19), para contrastar las políticas sociales con las de la etapa anterior, haciendo referencia a Latinoamérica: "Tenemos hoy privatización donde antes hubo intervención y regulación estatal; focalización en lugar de universalidad; compensación en vez de promoción; individualismo y particularismo clientelista como sustituto del ejercicio colectivo de derechos; combate a la pobreza extrema en lugar de desarrollo social".

Dentro de las acciones sociales del segundo período de gobierno de Pérez, también se ubica el denominado Megaproyecto Social, considerado la segunda fase de la política social y fue aprobado en el año 1992, mediante decreto No. 2.511. Estaba dirigido a atender la infraestructura en agua, salud, educación, vivienda, saneamiento ambiental, seguridad social y recreación. La inversión inicial a ejecutar durante los años 19921993 se calculó en Bs. 123 mil millones. Sin embargo, se redujo a la solicitud de un crédito adicional al Congreso Nacional por parte del Ministerio de Hacienda por la suma de Bs. 57,3 millardos, a ser financiados con recursos provenientes del proceso de privatización (Silva Michelena, 1999). 
En 1994, con la llegada al poder del Presidente Caldera, se diseña el Plan de Solidaridad Social, que sustituye al PEP e implica ligeros cambios en la estructura de los programas contenidos en el Plan antes mencionado. Tiene como objetivo la lucha contra la pobreza, a partir de la construcción de una economía sana que permita superar el desempleo, la remuneración insuficiente y las situaciones de miseria ${ }^{8}$. En ese mismo año se da a conocer el Plan de Recuperación y Estabilización Económica (PERE), que contiene un aparte dedicado al área social, denominado "La Estrategia de Acción Social", donde se incorpora gran parte de los programas sociales dados a conocer en el Plan de Solidaridad Social.

En 1996, se aplica en el país un nuevo programa de ajuste de naturaleza ortodoxa, luego de los intentos frustrados del presidente Caldera de diseñar una estrategia económica desvinculada del paradigma neoliberal. Sin embargo, la situación económica, donde se destaca la crisis financiera-bancaria, hace necesaria la concreción de un nuevo acuerdo con el FMI.

En el marco del nuevo programa de ajuste, denominado Agenda Venezuela, se diseñan los siguientes programas sociales conocidos como el Componente Social de la mencionada Agenda, en el que se destaca la diferenciación entre lo "económico" y lo "social" implícita en el programa: Subsidio Familiar; Dotación de Uniformes y Útiles Escolares; Programa Alimentario Escolar; Programa Alimentario Materno Infantil; Desayuno, Merienda y Comedores Escolares; Suministro de Medicamentos; Subsidio al Pasaje Estudiantil; Capacitación y Empleo Joven; Hogares y Multihogares de Cuidado Diario; Programa de Alimentos Estratégicos; Fondo de Fortalecimiento Social; Incremento de Pensiones de Vejez, Dotación de Material Médico-Quirúrgico y Atención Integral al Anciano.

El componente social de la Agenda Venezuela tiene como objetivo: "...compensar la caída del ingreso debido al largo período de recesión que afecta al aparato productivo desde hace dos décadas, y al programa de ajuste económico que el gobierno ve en la necesidad de acometer, con el fin de reestablecer los equilibrios macroeconómicos y crear un ambiente favorable al crecimiento sano de la economía nacional"(Márquez y Lima, 1999:91).

Estos programas sociales no difieren de manera significativa de los contemplados en el PEP. Puesto que se mantuvo el objetivo de obtener un impacto inmediato sobre la población más pobre, a través de subsidios directos (dinero, especies o servicios), que perseguían fortalecer el ingreso y evitar la caída de la calidad de vida. Sin embargo, es de resaltar las diferencias en los contextos en que se aplicaron. La Agenda Venezuela se aplicó en un clima de consenso social, lo que explica el asentimiento que tuvo en la población y, obviamente, no generó la resistencia social resultante de la aplicación del anterior proceso de ajuste (de 1989). Según Pulido de Briceño (1999:131), "la reacción popular fue de

8 De donde se desprende que el enfoque de política social que subyace puede resumirse en la conocida oración: "la mejor política social es una buena política económica". 
resignación", situación producida por la conjunción de dos procesos, por una parte, por la necesidad de las autoridades de tomar decisiones ante la magnitud de los desequilibrios macroeconómicos y; por otra parte, las condiciones reales de la situación socioeconómica incidieron en la aceptación por parte de la población.

Si se realiza un balance del impacto que tuvieron estos programas sociales sobre la población a la que fueron dirigidos (los sectores más vulnerables), destaca el deterioro de las condiciones de vida de la población, evidenciado en el incremento de los niveles de pobreza. Así, por ejemplo, Silva Michelena (1999: 9597) citando a Silva y Schliesser y Riutort (2001:44), exponen los siguientes datos:

- El poder adquisitivo de las familias venezolanas se deterioró aproxima damente en $71 \%$, entre 1980 y 1996.

- La tasa de desempleo aumentó de 6\% en 1993 a 12\% en 1997.

- El porcentaje de hogares en situación de pobreza se incrementó de $23,4 \%$ en 1992 a 58,5\% en 1997.

A manera de conclusión, podemos señalar que estos programas compensatorios, orientados hacia el corto plazo y a sectores delimitados de la sociedad, no cumplieron con los objetivos que se plantearon, dado que no paliaron los costos sociales del ajuste en la población. Por el contrario, el deterioro de las condiciones de vida se profundizó. Además, en lo que refiere a los logros económicos, los programas de ajuste han tenido limitaciones, evidencia de ello es, la persistencia de la crisis económica, con lo que los frutos del crecimiento económico tampoco se han derramado sobre la población.
Sin embargo, es de mencionar el planteamiento de López Maya y Lander (2000) sobre el tema. Para estos autores los indicadores socioeconómicos no permiten evaluar el impacto que, a nivel social, han tenido los planes de ajuste. Si bien confirman el deterioro de los indicadores mencionados, consideran que la interrupción de los planes de ajuste al poco tiempo de su inicio, demuestra la inconsistencia en su aplicación y puede distorsionar o prestar a diferentes interpretaciones los resultados obtenidos. Ello explica las razones por las cuales quienes realizan una apología de la perspectiva neoliberal, señalan que las cifras negativas de los indicadores socioeconómicos obtenidas durante los años de aplicación de los programas de ajuste son resultado de la inconsistencia con que se han aplicado estos programas. Mientras quienes se ubican en el extremo opuesto y demonizan el neoliberalismo, la lectura que realizan de las mismas cifras los lleva a concluir que son el resultado de lo perverso del modelo. En este sentido señalan : "... en rigor, para el caso venezolano, a partir de los indicadores socioeconómicos y de la experiencia turbulenta de los distintos intentos de implementación de política de ajuste, no es posible extraer conclusiones definitivas. Mientras en otros países de la región hay indicadores de resultados, como el abatimiento de la inflación o la recuperación de un crecimiento interanual sostenido, que permiten lecturas diversas según la perspectiva teórica del analista, en Venezuela los datos empíricos nos confrontan con una irrefutable situación de deterioro económico y social donde el agotamiento del 
viejo modelo como la incapacidad de las políticas de ajuste implementadas se conjugan" (López Maya y Lander, 2000: 187).

Sin embargo, llama la atención lo alarmante de algunas cifras que señalan los autores antes mencionados, tales como:

- La disminución del empleo formal público, de $22,67 \%$ en 1983 a $16,33 \%$ en 1998. El incremento del empleo del sector informal, de $41,30 \%$ en 1983 a 48,20\% en 1998 .

- Disminución del $42,95 \%$ del ingreso anual promedio entre 1979 y 1997 , es decir, de $\$ 5.345$ a $\$ 3.049$.

\section{Hacia la desarticulación de la política social y los programas sociales (1999-2001)}

A partir de 1999, con la llegada al poder del actual presidente Hugo Chávez Frías, quien gana las elecciones presidenciales efectuadas en diciembre de 1998 con una amplia mayoría, se comienzan a vislumbrar cambios en el ordenamiento socio-político de la sociedad. En el marco de su proyecto de refundación del país e instauración de la V República, se elabora y sanciona una nueva Constitución, la de 1999.

En lo que se refiere al modelo de desarrollo, destaca la inexistencia de la definición de las estrategias económicas y sociales a seguir, esto significa la ausencia de un proyecto de desarrollo. También explica por qué las acciones sociales diseñadas y puestas en práctica están desarticuladas de un proyecto de corto, mediano y/o largo plazo.

Como documento "oficial" que contiene los lineamientos de la política social se conoce "La Revolución Social en la V República. La Política Social de Venezuela. Cumbre Mundial de Desarrollo Social. Ginebra, Suiza", elaborado por el Ministerio de Salud y Desarrollo Social (MSDS), en el año 2000. En este documento se señala la necesidad de tener una visión equilibrada de lo social y lo económico, otorgándole igualdad de status a ambas esferas. En este marco, se plantea consolidar el capital humano y el capital social como vías para alcanzar el desarrollo económico y social.

En cuanto a los principios generales de la política social, la propuesta -fundamentada en una concepción humanista del Estado de Derecho- señala los siguientes: 1) La integración social, como mecanismo para incorporar la participación de la población en las decisiones de política pública social. 2) La corresponsabilidad, como instrumento a través del cual el ciudadano asume conjuntamente con el estado las decisiones que atañen a su participación en la vida pública. 3) La cohesión social, principio a promover para que los ciudadanos, respetando la diversidad, se involucren y ac-

9 El período analizado en esta sección abarca hasta noviembre de 2001, fecha de finalización del artículo. Sin embargo, es de aclarar que el 28 de septiembre de 2001, el Presidente de la República presentó el Plan realizar un análisis de la propuesta social. Estudio que quedará para una próxima investigación. 
cedan a la definición del proyecto de país. 4) La participación social, como estrategia que apunta a la apertura de los espacios públicos, de manera de distribuir el poder antes centrado en el estado. 5) La ciudadanización, como proceso para el reconocimiento de los deberes, derechos y garantías de los ciudadanos. 6) La progresividad, con la finalidad de jerarquizar las acciones sociales a ser ejecutadas en el corto, mediano y largo plazo. 7) La intersectorialidad, para que la gestión social abarque interrelacionadamente las distintas áreas que interviene. 8) La familia, como grupo social al cual -de acuerdo a lo establecido en la Constitución- se le otorgará protección global. 9) El desarrollo humano integral, como fin a alcanzar por la gestión social, es un proceso holístico y multidimensional, que hay que abordar interrelacionadamente (Ministerio de Salud y Desarrollo Social, 2000).

Según el documento referido, los programas que contempla la agenda social diseñada son: Familia, Vivienda, Hábitat, Ingreso, Emergencias Naturales y Sociales, Inclusión Social y Plan Bolívar 2000.

Sin embargo, en la práctica, el eje de la agenda social del actual gobierno se ha reducido a un dispositivo ejecutorio, el Plan Bolívar 2000 (CORDIPLAN, 1999), anunciado en febrero de 1999. Este se define como un Plan civíco-militar a ser ejecutado por la Fuerza Armada Nacional, el gobierno y la comunidad organiza- da, con el propósito de combatir la pobreza crítica y el cuadro social que vive el país ${ }^{10}$. Contempla una multiplicidad de intervenciones en diferentes áreas, desde salud, educación, desempleo, alimentación, hasta infraestructura y seguridad.

El Plan está dividido en tres (3) etapas: 1) Proyecto País, establece la provisión de asistencia para la población más necesitada; 2) Proyecto Patria, consiste en incorporar a los empleados públicos y desempleados a las actividades de atención a la sociedad, y a organizar a las comunidades para el trabajo productivo y, 3) Proyecto Nación, propone el desarrollo de proyectos estructurales a nivel socio-económico; así como, en el desarrollo de un proyecto de educación masiva.

Tal como se indica en la exposición de motivos, el Plan fue diseñado para enfrentar la crítica situación del país, expresada en los indicadores sociales que muestra: $80 \%$ de pobreza (39\%de pobreza extrema y $14 \%$ de indigencia), $15 \%$ de desempleo, $50 \%$ de empleo informal, $37 \%$ de desnutrición infantil, 21 por mil mortalidad infantil y $30 \%$ de deserción escolar.

Es necesario destacar que el Plan Bolívar 2000, por su propia naturaleza contingente, altera los principios que habían definido la agenda social propuesta y el programa de gobierno del presidente; asignándole a la política social características similares a la de los anteriores gobiernos: un carácter asistencialista, compensatorio y focalizado. 
En efecto, en el Capítulo II titulado "Equilibrio Social: Hacia una sociedad justa" de su programa de gobierno titulado "La propuesta de Hugo Chávez... para transformar a Venezuela. Una revolución democrática" (Chávez Frías, 1998), se plantea la estrategia social al país, hace referencia a la elaboración de una política social que define como global y sectorial, equitativa, integral, estructural, orientada a apoyar los esfuerzos de las familias, orientada a solucionar los problemas de las familias y con carácter de integración social. Éstos elementos definen una concepción de política social que le distanciaría de aquellas implantadas por los gobiernos anteriores. Sin embargo, se advierte una incongruencia entre lo propuesto en el programa de gobierno y el plan social que ejecuta actualmente, es decir, entre la propuesta discursiva y las realizaciones a partir de los planes propuestos.

Además, es relevante tener en consideración que se ha presentado a la opinión pública la continuación del Plan Bolívar en el año en curso, bajo la denominación de Plan Bolívar 2001, lo que es indicativo de que se tiene previsto continuar interviniendo la cuestión social a través de planes coyunturales y en este caso anuales, sin vinculación con una imagen objetivo a alcanzar en lo mediato. En otras palabras, sin tomar en cuenta acciones que apunten a provocar cambios estructurales, a invertir en lo social, a fortalecer el recurso humano.
En relación con las instituciones destinadas a la prestación de servicios sociales llama la atención que, en el marco de la reestructuración de la Administración Pública, se eliminó el Ministerio de la Familia, ente encargado de la coordinación de las actividades de la política social, y se creó el Ministerio de Salud y Desarrollo Social; lo que da una visión de la concepción y de la importancia que se le asigna al desarrollo social. Se adscribió a este nuevo Ministerio el Fondo Unico Social $^{11}$, ente encargado de la administración de los recursos para la ejecución de algunos programas sociales que aún se mantienen de la administración anterior y otros de nuevo diseño.

Entre otras medidas sociales, se destacan las que se mencionan a continuación:

- El Acuerdo de Caracas, firmado con Cuba en octubre del 2000, mediante el cual el mencionado país suministra cooperación a Venezuela en las áreas de salud, deportes y agrícola a cambio de petróleo. En su marco, se envían pacientes venezolanos a recibir tratamiento médico especializado en instituciones de salud pública cubana. Ello ante el deterioro de la prestación del servicio de salud en los centros hospitalarios del país.

- La creación del Banco del Pueblo, organización financiera del Estado que atiende a un segmento específico, a

11 Creado según Decreto No. 300, aparecido en la Gaceta Oficial de la República Bolivariana de Venezuela No. 36.781, de fecha 07-09-99, como un ente presupuestario dependiente del Ministerio de Salud y Desarrollo Social, con el objetivo de concentrar la captación y administración de recursos destinados a la ejecución de programas orientados al desarrollo social. 
los microempresarios. Inició sus actividades el 15-09-99.

- La creación del Banco de la Mujer, con el objetivo de abrir una plaza financiera para otorgar créditos a la población del sexo femenino excluida del sistema bancario, promover la microempresa y coadyuvar a la superación de la pobreza. Forma parte de la política gubernamental que se orienta a brindar mayor atención a los sectores productivos marginados por años. Inició sus operaciones el 17-09-01.

- El anuncio del presidente realizado el 22-02-01, referido al hecho de que la banca pública (Banco del Pueblo, Banco de la Mujer y el Banco Industrial de Venezuela) no cobrará intereses a los microcréditos otorgados a las clases populares, cuyas acreencias sean menores al millón de bolívares.

Estas acciones, han sido anunciadas en diferentes fechas y sin vinculación con una estrategia global de desarrollo; así como tampoco se articulan, tal como lo hemos expresado, a la definición de los lineamientos de la política social a seguir. Obedecen, más bien, a la solución de problemas sociales coyunturales y muy puntuales, que si bien demandan respuestas inmediatas, no pueden agotar los esfuerzos programáticos de una política social. De esta panorámica, se deduce que no existe una definición de política social integral, las acciones sociales instrumentadas están desarticuladas, pues el horizonte temporal en el cual se ejecutan es el presente, el inmediatismo; crítica que se aplica a su vez, a la concepción de los Planes Bolívar 2000 y 2001.
Con respecto a la seguridad social, no puede dejar de mencionarse la situación de indefinición reinante. Esta se ha mantenido en vacatio legis desde enero de 1999. En un primer momento, ante la aprobación de la Ley de Seguridad Social en octubre de 1998 (por el presidente Caldera dos meses antes de dejar el cargo) se aplicó una vacatio legis de un año, hasta el mes de enero del año 2000. Con el cambio de gobierno este período se prolongó hasta la revisión de la Ley, a tal efecto se nombró una Comisión Presidencial de Seguridad Social para que diseñara un nuevo proyecto de Ley de Seguridad Social, el cual recientemente fue entregado al presidente de la República para su discusión y aprobación.

Si se realiza un balance social de estos dos años, se observa que las condiciones de vida de la población han continuado deteriorándose. En este contexto contrasta que la popularidad del presidente se mantiene elevada, sobretodo en los estratos socioeconómicos bajos. Cuando asumió la presidencia su popularidad alcanzaba a $91,9 \%$, y a comienzos de este año 2001, en el mes de febrero, alcanza a $62,2 \%$, si se desagrega este dato se obtiene que en los estratos bajos ( $D$ y $E$ ), que representan el $81 \%$ de la población, el $63,2 \%$ y el $67,5 \%$, respectivamente, consideran la ejecutoria del presidente como muy buena, buena y regular hacia buena. Mientras que el porcentaje de población que cataloga su labor como muy mala, mala y regular hacia mala, alcanza al $36,1 \%$, y se ubica en los estratos socioeconómicos altos (Datanálisis, 2001).

La reflexión en torno a estos datos lleva a las siguientes consideraciones: 
Por una parte, que uno de los componentes explicativos de la aceptación del presidente y de la calificación positiva de su gestión tiene su base en el sistema clientelar de relaciones que desarrolla a través de las acciones sociales que instrumenta, lo que nos permite decir que estamos en presencia de una legitimidad por dádivas ${ }^{12} \mathrm{y}$, en consecuencia, de un régimen con un alto componente neopopulista $^{13}$.

Por otra parte, que la población de los estratos bajos preservan una representación mesiánica del presidente que permite mantengan su confianza en su capacidad para resolver los problemas que confrontan. Además, porque las expectativas generadas en torno a su figura como constructor de un nuevo orden, que recogió en sus discursos ante el clima de desencanto, desarraigo, incertidumbre presente en la sociedad venezolana, continúan presentes.

El presidente elabora una construcción simbólica anclada en elementos de la cultura política y del imaginario popular del venezolano (Bermúdez y Martínez, 2000). El imaginario colectivo que ha construido (referentes sociales, identidades políticas, códigos interpretativos), basado en invocaciones del pasado he- roico y de la religiosidad popular cristiana, creó nuevas identificaciones y formas de hacer política con las cuales la sociedad se siente representada y, por tanto, le hacen mantener la esperanza en torno a la venida de un futuro mejor.

\section{Conclusiones}

La política social diseñada desde 1989, en el marco de los cambios acontecidos por la crisis generalizada de la sociedad que delineó como salida la implantación de procesos de ajuste estructural, se caracteriza por ser parte de una estrategia que apunta a intervenir los desequilibrios coyunturales ocasionados por el ajuste económico. Este tipo de política está desvinculada de una estrategia de desarrollo de mediano y largo plazo. La compensación de los efectos perversos de la política económica persigue, a nivel sociopolítico, la posibilidad de mantener un clima de negociación y armonía social ante los cambios propuestos para mantener la legitimidad del régimen. De acuerdo con este marco de referencia, la política social cumplió el papel de paliar la crisis de integración social producida por la pérdida de la capacidad del Estado para manejar el consenso y la legitimidad,

12 Se habla de legitimidad por dádivas cuando la lealtad de la población al orden establecido de pende de los beneficios que reciben del Estado o de quien detenta el poder político de forma directa (Serrano Gómez, 1994).

13 El neopopulismo refiere a aquellos regímenes en los cuales el cuerpo intermedio de canales (partidos políticos u otras instituciones) y redes sociales de mediación entre el Estado y la sociedad, queda sustituido por figuras carismáticas. Los líderes, en el marco de los vínculos emotivos que mantienen con el pueblo, interpretan y canalizan sus demandas. A diferencia del llamado populismo, en el que entre el Estado y la sociedad se desarrollan organizaciones (como partidos políticos u otras instituciones) que actúan como canales de mediación de las demandas de la población. 
pues el Estado no podía seguir satisfaciendo, simultáneamente, las demandas de la economía que permiten la reproducción del capital y las demandas de la sociedad civil con las cuales asegura la lealtad de las masas y la legitimidad.

Las acciones sociales implantadas desde 1999 también mantienen un carácter coyuntural, compensatorio y focalizado, desarticulado de un modelo de desarrollo económico social. Pero, en este período resalta la ausencia de lineamientos, programas y proyectos que establezcan las prioridades que la critica situación social reclama, presentándose una situación social de incertidumbre. En este caso, la figura carismática y la representación mesiánica que la población tiene de la figura del presidente, además de las relaciones clientelares que establece a través de la distribución de bienes y servicios, explican el reconocimiento de la población a la gestión gubernamental y las expectativas de cambio y elevación de las condiciones de vida que se han dibujado en el contexto de la realidad sociopolítica y económica.

\section{Bibliografía Citada}

Arenas, Ender (1992), "La crisis de febrero: un análisis de sus múltiples lecturas o cómo analizar el 4 febrero y que no nos den un golpe mientras lo hacemos", Estudios de Coyuntura, No. 6, Venezuela, Universidad del ZuliaFCES, pp. 9-25.

Bermúdez, Emilia y Martínez, Gildardo (2000), "Hugo Chávez: La articulación de un sentido para la acción colectiva", $\mathbf{R e}$ vista Espacio Abierto, Vol. 9, No. 1, Venezuela, Asociación Venezolana de Sociología-International Sociological Association, pp. 53-77.

Chávez Frías, Hugo (1998), La propuesta de Hugo Chávez... para transformar a Venezuela. Una revolución democrática. (Http://www.cordiplan.gov.ve/ prog-gob/prog_gob.htm) (Fecha de consulta: 12-06-2000).

CORDIPLAN (1999), Proyecto Bolívar 2000. (Http://cordiplan.gov.ve/ Prog-B-2000.htm) (Fecha de consulta: 16-11-1999).

CORDIPLAN (1990), VIII Plan de la Nación 1990-1993. Caracas.

Datanálisis (2001), "Popularidad de Chávez creció de 55,9 a 62,7 por ciento". Diario La Verdad. Pág. A-8, 02-03-2001.

González, Lissette (1996), La política social en Venezuela. Curso de Formación Sociopolítica, No. 35, Caracas, Fundación Centro Gumilla.

Habermas, Jürgen (1975), Crisis de legitima ción en el capitalismo tardío. Argentina, Amorrortu Editores.

Kornblith, Miriam (1994), "La crisis del sistema político venezolano". Revista Nueva Sociedad, No. 134, Venezuela. pp. 83-99.

Lacabana, Miguel y Maingón, Thaís (1997), "La focalización: políticas sociales "estructuralmente ajustadas". Cuadernos del Cendes, Año 14. No. 34, Caracas, Centro de Estudios del Desarrollo de la Universidad Central de Venezuela, pp. 193-218.

López Maya, Margarita y Lander, Luis E. (2000), "Ajustes, costos sociales y agenda de los pobres en Venezuela: 1984-1998". Revista Venezolana de Economía y Ciencias Sociales, Vol. 6, No. 3, Caracas, Instituto de Investigaciones Económicas y Sociales Dr. Rodolfo Quintero, Universidad Central de Venezuela, pp. 185-206. 
Luhmann, Niklas (1990), Sociedad y sistema: la ambición de la teoría. Barcelona, Editorial Paidós.

Márquez, Trino y Lima, Boris (1999), "La política social: pasado reciente y futuro inmediato". Revista Venezolana de Economía y Ciencias Sociales, Vol. 5, No. 1, Caracas, Instituto de Investigaciones Económicas y Sociales Dr. Rodolfo Quintero, Universidad Central de Venezuela, pp. 89-110.

Ministerio de Salud y Desarrollo Social (2000), La revoluciòn social en la $V$ Repùblica. La politica social de Venezuela. Cumbre Mundial de Desarrollo social. Ginebra, Suiza. Mimeografiado. 110 págs.

Piñango, Ramón (1999), "La política social en las últimas dos décadas", en: Política social: exclusión y equidad en Venezuela durante los años Noventa, Alvarez y otros (Coordinadores), Venezuela, Fonvis-Cendes- Ildis-Nueva Sociedad, pp. 115-122.

Portillo, Gustavo (1998), La crisis en tiempo de democracia (1958-1960 y 1984). Caracas, Consejo de Desarrollo Científico y Humanístico de la Universidad Central de Venezuela.

Pulido de Briceño, Mercedes (1999), "El impacto de los programas sociales. Balance y perspectivas", en: Política social: exclusión y equidad en Venezuela durante los años noventa, Alvarez y otros (Coordinadores), Venezuela, Fonvis- Cendes- Ildis-Nueva Sociedad, pp. 131-139.
Rey, Juan Carlos (1989), El futuro de la democracia en Venezuela. Colección Idea, Caracas.

Riutort, Matías (2001), "Inflación, desempleo y pobreza", en: El camino por recorrer, Vol. 2. Documentos del Proyecto Pobreza, Caracas, Universidad Católica Andrés Bello-Asociación Civil para la Promoción de Estudios Sociales, pp. 41-53.

Serrano Gómez, Enrique (1994), Legitimación y racionalización. Weber y Habermas: la dimensión normativa de un orden secularizado. España, Anthropos-Universidad Autónoma Metropolitana.

Silva Michelena, Héctor (1999), "La política social en Venezuela durante los años ochenta y noventa", en: Política social: exclusión y equidad en Venezuela durante los años noventa, Alvarez y otros (Coordinadores), Venezuela, Fonvis-Cendes- Ildis- Nueva Sociedad, pp. 85- 114.

Vilas, Carlos (1995), "Después del ajuste: la política social entre el Estado y el mercado"; en: Estado y políticas sociales después del ajuste, Carlos Vilas (Coord.), Venezuela, UNAM-Nueva Sociedad.

Vivancos, Francisco y España, Luis Pedro (1993), La crisis que nos falta por recorrer. ILDIS-Editorial Nueva Sociedad, Venezuela. 